\title{
S3 Symmetric Scotogenic Model for Realistic Neutrino Mixing
}

\author{
Soumita Pramanick \\ National Centre for Nuclear Research, Pasteura 7, 02-093 Warsaw, Poland
}

\begin{abstract}
In a $S 3 \times Z_{2}$ framework, realistic neutrino mixing was obtained radiatively at one-loop level. When maximal mixing occurs between the two right-handed neutrinos present in the model, one can get the form of the left-handed Majorana neutrino mass matrix for $\theta_{13}=0, \theta_{23}=\pi / 4$ and solar mixing of any values corresponding to the Tribimaximal (TBM), Bimaximal (BM) and Golden Ratio (GR) mixing scenarios in this set-up. Once we tweak the maximal mixing between the two right-handed neutrinos, we get non-zero $\theta_{13}$, deviation of $\theta_{23}$ from $\pi / 4$ and small corrections to solar mixing. This scotogenic model also has two inert $S U(2)_{L}$ doublet scalars odd under $Z_{2}$ the lightest of which can be dark matter.
\end{abstract}

Keywords: neutrino mass and mixing, radiative model, discrete flavour symmetry S3

DOI: 10.31526/ACP.BSM-2021.27

\section{INTRODUCTION}

This talk is based ${ }^{1}$ on [1]. The Pontecorvo, Maki, Nakagawa, Sakata - PMNS - matrix, relating the neutrino mass and flavour eigenstates is given by:

$$
U=\left(\begin{array}{ccc}
c_{12} c_{13} & s_{12} c_{13} & -s_{13} e^{-i \delta} \\
-c_{23} s_{12}+s_{23} s_{13} c_{12} e^{i \delta} & c_{23} c_{12}+s_{23} s_{13} s_{12} e^{i \delta} & -s_{23} c_{13} \\
-s_{23} s_{12}-c_{23} s_{13} c_{12} e^{i \delta} & -s_{23} c_{12}+c_{23} s_{13} s_{12} e^{i \delta} & c_{23} c_{13}
\end{array}\right)
$$

with $c_{i j}=\cos \theta_{i j}$ and $s_{i j}=\sin \theta_{i j}$. If we put $\theta_{13}=0$ and $\theta_{23}=\pi / 4$, the PMNS matrix in Eq. (1) simply becomes:

$$
U^{0}=\left(\begin{array}{ccc}
\cos \theta_{12}^{0} & \sin \theta_{12}^{0} & 0 \\
-\frac{\sin \theta_{12}^{0}}{\sqrt{2}} & \frac{\cos \theta_{12}^{0}}{\sqrt{2}} & -\frac{1}{\sqrt{2}} \\
-\frac{\sin \theta_{12}^{0}}{\sqrt{2}} & \frac{\cos \theta_{12}^{0}}{\sqrt{2}} & \frac{1}{\sqrt{2}}
\end{array}\right) .
$$

In 2012, the short-baseline reactor anti-neutrino experiments [2] discovered non-zero $\theta_{13}$, before which models were constructed with $\theta_{13}=0$ and $\theta_{23}=\pi / 4$ and $\theta_{12}$ varying to the specific values mentioned in Table 1 to yield the Tribimaximal (TBM), Bimaximal (BM) and Golden Ratio (GR) mixing patterns. These TBM, BM and GR scenarios are also called together as popular lepton mixings and have the structure of the mixing as in Eq. (2). The left-handed Majorana neutrino mass matrix in flavour basis for the popular lepton mixings is thus given by:

$$
M_{v L}^{\text {flavour }}=U^{0} M_{v L}^{\text {mass }} U^{0 T}=\left(\begin{array}{lll}
a & c & c \\
c & b & d \\
c & d & b
\end{array}\right),
$$

where $M_{v L}^{\text {mass }} \equiv\left(m_{1}^{0}, m_{2}^{0}, m_{3}^{0}\right)$ and ${ }^{2}$

$$
\begin{aligned}
a & =m_{1} \cos ^{2} \theta_{12}^{0}+m_{2} \sin ^{2} \theta_{12}^{0}, \\
b & =\frac{1}{2}\left(m_{1} \sin ^{2} \theta_{12}^{0}+m_{2} \cos ^{2} \theta_{12}^{0}+m_{3}\right), \\
c & =\frac{1}{2 \sqrt{2}} \sin 2 \theta_{12}^{0}\left(m_{2}-m_{1}\right), \\
d & =\frac{1}{2}\left(m_{1} \sin ^{2} \theta_{12}^{0}+m_{2} \cos ^{2} \theta_{12}^{0}-m_{3}\right) .
\end{aligned}
$$

Here,

$$
\tan 2 \theta_{12}^{0}=\frac{2 \sqrt{2} c}{b+d-a}
$$

\footnotetext{
${ }^{1}$ The paper [1] on which this talk is based on was written during my post-doctoral research at Harish-Chandra Research Institute, Allahabad, India.

${ }^{2}$ The three neutrino mass eigenstates viz. $m_{1}^{0}, m_{2}^{0}$ and $m_{3}^{0}$ are non-degenerate.
} 


\begin{tabular}{cccc}
\hline Model & TBM & BM & GR \\
\hline$\theta_{12}^{0}$ & $35.3^{\circ}$ & $45.0^{\circ}$ & $31.7^{\circ}$ \\
\hline
\end{tabular}

TABLE 1: Solar mixing angle values for TBM, BM, and GR.

The quantities $a, b, c$ and $d$ has to be non-zero for neutrino masses to be non-degenerate and realistic.

The $3 \sigma$ global fits of the neutrino mixing angles [3, 4] are:

$$
\begin{aligned}
& \theta_{12}=(31.42-36.05)^{\circ}, \\
& \theta_{23}=(40.3-51.5)^{\circ}, \\
& \theta_{13}=(8.09-8.98)^{\circ} .
\end{aligned}
$$

They clearly disagree with the popular lepton mixing scenarios such as TBM, BM and GR. Therefore, in order to make the neutrino mixings to be realistic, one has to depart from the structure of the left-handed Majorana neutrino mass matrix ${ }^{3}$ in Eq. (3).

In this work we consider a $S 3 \times Z_{2}$ setup ${ }^{4}$ to generate the neutrino mixings in agreement with the neutrino oscillation data radiatively at one-loop level. The model contains two $Z_{2}$ odd right-handed neutrinos which when mixed maximally can yield the structure of the left-handed Majorana mass matrix specific to $\theta_{13}=0$ and $\theta_{23}=\pi / 4$ as shown in Eq. (3). A slight deviation from this maximal mixing in the right-handed neutrino sector can yield the non-zero $\theta_{13}$, deviation of $\theta_{23}$ from $\pi / 4$ and small corrections to the solar mixing angle $\theta_{12}$ in one stroke as required to be in agreement with the neutrino oscillation data. The model also has two $Z_{2}$ odd $S U(2)_{L}$ doublet scalars $\eta_{i} \equiv\left(\eta_{i}^{+}, \eta_{i}^{0}\right)^{T},(i=1,2)$ transforming as a doublet under S3. The lightest among these two inert $S U(2)_{L}$ doublet scalars $\eta_{i}$ can be a dark matter candidate.

In the scalar sector two more $S U(2)_{L}$ doublet scalars $\Phi_{j} \equiv\left(\phi_{i}^{+}, \phi_{i}^{0}\right),(j=1,2)$ forming a $S 3$ doublet are present that are even under $Z_{2}$. In the lepton sector, the left-handed $S U(2)_{L}$ doublet leptons $L_{\zeta L} \equiv\left(\nu_{\zeta}, \zeta^{-}\right)_{L}^{T}$ with $\zeta=e, \mu, \tau$ are present among which $L_{\mu}$ and $L_{\tau}$ form a doublet under $S 3$ while $L_{e}$ is a singlet under $S 3$. All the fields are even under $Z_{2}$ except the right-handed neutrinos $N_{\alpha R},(\alpha=1,2)$ and $\eta_{i},(i=1,2)$. After spontaneous symmetry breaking (SSB), $\eta_{i}$ being $Z_{2}$ odd does not acquire vev but $\Phi_{j}$ gets vev i.e., $\left\langle\Phi_{j}\right\rangle=v_{j},(j=1,2)$. The quantum numbers of all the fields present in the model can be found in Table 2 . Here we consider the

\begin{tabular}{|c|c|c|c|}
\hline Leptons & $S U(2)_{L}$ & S3 & $Z_{2}$ \\
\hline$L_{e_{L}} \equiv\left(\begin{array}{ll}v_{e} & e^{-}\end{array}\right)_{L}$ & 2 & 1 & 1 \\
\hline$L_{\zeta_{L}} \equiv\left(\begin{array}{ll}v_{\mu} & \mu^{-} \\
v_{\tau} & \tau^{-}\end{array}\right)_{L}$ & 2 & 2 & 1 \\
\hline$N_{\alpha R} \equiv\left(\begin{array}{l}N_{1 R} \\
N_{2 R}\end{array}\right)$ & 1 & 2 & -1 \\
\hline Scalars & $S U(2)_{L}$ & S3 & $Z_{2}$ \\
\hline$\Phi \equiv\left(\begin{array}{ll}\phi_{1}^{+} & \phi_{1}^{0} \\
\phi_{2}^{+} & \phi_{2}^{0}\end{array}\right)$ & 2 & 2 & 1 \\
\hline$\eta \equiv\left(\begin{array}{ll}\eta_{1}^{+} & \eta_{1}^{0} \\
\eta_{2}^{+} & \eta_{2}^{0}\end{array}\right)$ & 2 & 2 & -1 \\
\hline
\end{tabular}
neutrino sector only and work in a basis in which the charged lepton mass matrix is diagonal and the whole mixing comes from the neutrinos.

TABLE 2: Particle content of the model with their respective properties under the symmetries of the model. We consider the neutrino sector only.

\footnotetext{
${ }^{3}$ Prior to this such enterprises had also been pursued in [5, 6]. In [6] a similar scotogenic model at one-loop level for realistic neutrino mixings based on $A 4$ symmetry can be found. A detailed analysis of the vacuum expectation value (vev) structures of the scalars present in [6] in the context of alignment was done in [7].

${ }^{4}$ In [1] detailed discussion about $S 3$ flavour symmetry can be found.
} 


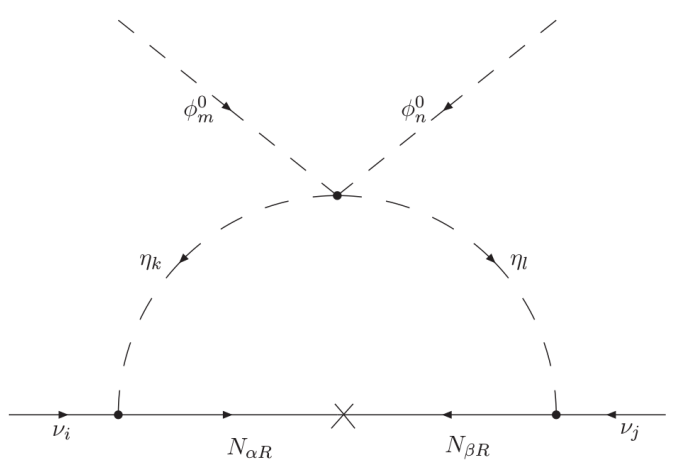

FIGURE 1: Neutrino mass at one-loop in a scotogenic $S 3 \times Z_{2}$ setup.

\section{THE MODEL}

The neutrino mass at one-loop level can be given by Fig. (1). The relevant terms ${ }^{5}$ of the $S 3 \times Z_{2}$ invariant scalar potential that can contribute to the left-handed Majorana neutrino mass matrix via the four-point scalar vertex in Fig. (1):

$$
\begin{aligned}
V_{\text {relevant }} & \supset \lambda_{1}\left[\left\{\left(\eta_{2}^{\dagger} \phi_{2}+\eta_{1}^{\dagger} \phi_{1}\right)^{2}\right\}+\text { h.c. }\right]+\lambda_{2}\left[\left\{\left(\eta_{2}^{\dagger} \phi_{2}-\eta_{1}^{\dagger} \phi_{1}\right)^{2}\right\}+\text { h.c. }\right] \\
& +\lambda_{3}\left[\left\{\left(\eta_{1}^{\dagger} \phi_{2}\right)\left(\eta_{2}^{\dagger} \phi_{1}\right)+\left(\eta_{2}^{\dagger} \phi_{1}\right)\left(\eta_{1}^{\dagger} \phi_{2}\right)\right\}+\text { h.c. }\right] .
\end{aligned}
$$

The couplings $\lambda_{j}(j=1,2,3)$ are real.

At each of the vertices in Fig. 1 all the symmetries are conserved. The $S 3 \times Z_{2}$ invariant Yukawa terms are:

$$
\mathscr{L}_{\text {Yukawa }}=y_{1}\left[\left(\bar{N}_{2 R} \eta_{2}^{0}+\bar{N}_{1 R} \eta_{1}^{0}\right) v_{e}\right]+y_{2}\left[\left(\bar{N}_{1 R} \eta_{2}^{0}\right) v_{\tau}+\left(\bar{N}_{2 R} \eta_{1}^{0}\right) v_{\mu}\right]+\text { h.c. }
$$

The $S 3 \times Z_{2}$ conserving right-handed neutrino direct mass terms:

$$
\mathscr{L}_{\text {right-handed neutrinos }}=\frac{1}{2} m_{R_{12}}\left[N_{1 R}^{T} C^{-1} N_{2 R}+N_{2 R}^{T} C^{-1} N_{1 R}\right] .
$$

If $S 3$ is conserved then one gets only non-zero off-diagonal terms in the right-handed Majorana neutrino mass matrix. The $S 3$ symmetry is broken softly by ${ }^{6}$ :

$$
\mathscr{L}_{\text {soft }}=\frac{1}{2}\left[m_{R_{11}} N_{1 R}^{T} C^{-1} N_{1 R}+m_{R_{22}} N_{2 R}^{T} C^{-1} N_{2 R}\right],
$$

to obtain non-zero diagonal terms in the right-handed Majorana neutrino mass matrix and write it as ${ }^{7}$ :

$$
M_{v_{R}}=\frac{1}{2}\left(\begin{array}{ll}
m_{R_{11}} & m_{R_{12}} \\
m_{R_{12}} & m_{R_{22}}
\end{array}\right) .
$$

The $Z_{2}$ symmetry in the model is responsible for stabilizing the dark matter candidate. We have $Z_{2}$ odd fields $\eta_{i},(i=1,2)$ and $N_{\alpha R},(\alpha=1,2)$ in our model. The right-handed neutrinos $N_{\alpha R}$ are chosen to be heavier than $\eta_{i}$. Thus the lightest of the $\eta_{i}$ scalars can behave as a dark matter candidate ${ }^{8}$.

Next we briefly discuss the contribution coming from the loop in Fig. (1) to the left-handed Majorana neutrino mass matrix [8]. For simplicity, let $\lambda$ represent commonly all the quartic couplings i.e., $\lambda_{1}, \lambda_{2}$ and $\lambda_{3}$ present in the $S 3 \times Z_{2}$ conserving scalar potential in Eq. (7). We also neglect the mass splittings between $\eta_{1}$ and $\eta_{2}$ and define $m_{0}$ as their common mass. If $\eta_{R j}$ and $\eta_{I j}$ be the real and imaginary parts of the $\eta_{j}^{0}$, then generally one can have the mass difference between $\eta_{R j}$ and $\eta_{I j}$ to be proportional to $\lambda v_{j}$. Taking $m_{R}$ to be the average mass of $N_{1 R}$ and $N_{2 R}$ and defining $z \equiv \frac{m_{R}^{2}}{m_{0}^{2}}$ we can write the second diagonal element of $M_{v_{L}}^{f l a v o u r}$ as $^{9}$ :

$$
\left(M_{v_{L}}^{\text {flavour }}\right)_{22}=\lambda \frac{v_{m} v_{n}}{8 \pi^{2}} \frac{y_{2}^{2}}{m_{R_{22}}}[\ln z-1] \text {. }
$$

\footnotetext{
${ }^{5}$ The total $S 3 \times Z_{2}$ invariant scalar potential is given in [1] out of which only the $\left(\eta^{\dagger} \phi\right)\left(\eta^{\dagger} \phi\right)$ terms contribute to the neutrino mass matrix as at the four-point scalar vertex in Fig. (1), two $\eta$ fields are created and two $\phi$ fields are annihilated.

${ }^{6}$ The $S 3$ symmetry is broken softly at the scale at which the right-handed neutrinos acquire their mass.

${ }^{7}$ The symmetric nature of the matrix in Eq. (11) is due to its Majorana nature.

${ }^{8}$ It might appear that the $S 3$ symmetry will cause the $\eta_{i}$ fields to be equal in masses, but since the $S 3$ symmetry is softly broken at the scale in which $N_{\alpha R}$ gets mass, a small mass splitting between the two $\eta_{i}$ fields can be achieved.

${ }^{9}$ For detailed discussion, see [1]
} 
when $m_{R}^{2}>>m_{0}^{2}$. From Eq. (8), one can notice that $v_{\mu}$ couples only with $N_{2 R}$ at both the Yukawa vertices in Fig. (1), $y_{2}$ being the corresponding Yukawa coupling. Thus in Eq. (12) we only have contributions from $y_{2}$ and $m_{R_{22}}$ of Eq. (11). Similarly the $\left(M_{v_{L}}^{\text {flavour }}\right)_{33}$ can also be read off by replacing $m_{R_{22}}$ by $m_{R_{11}}$ in the expression of $\left(M_{v_{L}}^{\text {flavour }}\right)_{22}$ in Eq. (12). For the (2,3) off-diagonal entry of $M_{v_{L}}^{\text {flavour }}$, we have $v_{\mu}$ at one Dirac Yukawa vertex and $v_{\tau}$ at the other Dirac Yukawa vertex of Fig. (1) that couple to $N_{2 R}$ and $N_{1 R}$ respectively, $y_{2}$ being the Yukawa coupling at both the vertices. Thus $\left(M_{v_{L}}^{\text {flavour }}\right)_{23}$ will depend on $m_{R_{12}}$ together with $m_{R_{11}}$ and $m_{R_{22}}$ of Eq. (11). Thus,

$$
\left(M_{v_{L}}^{\text {flavour }}\right)_{23}=\lambda \frac{v_{m} v_{n}}{8 \pi^{2}} \frac{y_{2}^{2} m_{R_{12}}}{m_{R_{11}} m_{R_{22}}}[\ln z-1] .
$$

Similarly we can get the other entries of $M_{\nu_{L}}^{\text {flavour }}$. In order to make the equations look simpler we can absorb everything else in the expressions of $\left(M_{v_{L}}^{\text {flavour }}\right)_{\alpha \beta},(\alpha, \beta=1,2,3)$ except the vevs, the Yukawa couplings and the quartic couplings in some loopcontributing factors $r_{\alpha \beta}$ given by:

$$
\begin{aligned}
r_{11} & \equiv \frac{1}{8 \pi^{2} m_{R_{11}}}[\ln z-1] \\
r_{22} & \equiv \frac{1}{8 \pi^{2} m_{R_{22}}}[\ln z-1], \\
r_{12} & \equiv \frac{m_{R_{12}}}{8 \pi^{2} m_{R_{11}} m_{R_{22}}}[\ln z-1] .
\end{aligned}
$$

for the expressions of elements of $M_{v_{L}}^{\text {flavour }}$ in Eqs. (12) and (13).

The left-handed Majorana neutrino mass matrix obtained in this setup:

$$
M_{v_{L}}^{\text {flavour }}=\left(\begin{array}{lll}
\chi_{1} & \chi_{4} & \chi_{5} \\
\chi_{4} & \chi_{2} & \chi_{6} \\
\chi_{5} & \chi_{6} & \chi_{3}
\end{array}\right)
$$

with,

$$
\begin{aligned}
\chi_{1} & \equiv y_{1}^{2}\left[4 r_{12} v_{1} v_{2}\left(\lambda_{3}+\lambda_{1}-\lambda_{2}\right)+\left(r_{11} v_{1}^{2}+r_{22} v_{2}^{2}\right)\left(\lambda_{1}+\lambda_{2}\right)\right] \\
\chi_{2} & \equiv y_{2}^{2}\left[r_{22}\left(\lambda_{1}+\lambda_{2}\right) v_{1}^{2}\right] \\
\chi_{3} & \equiv y_{2}^{2}\left[r_{11}\left(\lambda_{1}+\lambda_{2}\right) v_{2}^{2}\right] \\
\chi_{4} & \equiv y_{1} y_{2}\left[r_{12}\left(\lambda_{1}+\lambda_{2}\right) v_{1}^{2}+2 r_{22}\left(\lambda_{3}+\lambda_{1}-\lambda_{2}\right) v_{1} v_{2}\right] \\
\chi_{5} & \equiv y_{1} y_{2}\left[r_{12}\left(\lambda_{1}+\lambda_{2}\right) v_{2}^{2}+2 r_{11}\left(\lambda_{3}+\lambda_{1}-\lambda_{2}\right) v_{1} v_{2}\right] \\
\chi_{6} & \equiv y_{2}^{2}\left[2 r_{12}\left(\lambda_{3}+\lambda_{1}-\lambda_{2}\right) v_{1} v_{2}\right]
\end{aligned}
$$

and $\left\langle\Phi_{j}\right\rangle \equiv v_{j}$ where $(j=1,2)$. If $\chi_{1} \neq \chi_{2}=\chi_{3}$ and $\chi_{4}=\chi_{5}$, we get the structure of neutrino mass matrix in Eq. (3) i.e., the one specific to $\theta_{13}=0, \theta_{23}=\pi / 4$. One can indeed get this for $v_{1}=v_{2}$ and $r_{11}=r_{22}=r$. Now $r_{11}=r_{22}=r$ basically means $m_{R_{11}}=m_{R_{22}}$ in Eq. (11) which in its turn indicate maximal mixing between the two right-handed neutrino states $N_{1 R}$ and $N_{2 R}$. Setting $r_{11}=r_{22}=r$ and $v_{1}=v_{2}$ in Eq. (15) leads to:

$$
M_{v_{L}}^{\text {flavour }}=v^{2}\left(\begin{array}{ccc}
y_{1}^{2}\left[4 r_{12} \lambda_{123}+2 r \lambda_{12}\right] & y_{1} y_{2}\left[r_{12} \lambda_{12}+2 r \lambda_{123}\right] & y_{1} y_{2}\left[r_{12} \lambda_{12}+2 r \lambda_{123}\right] \\
y_{1} y_{2}\left[r_{12} \lambda_{12}+2 r \lambda_{123}\right] & y_{2}^{2} r \lambda_{12} & y_{2}^{2}\left(2 r_{12} \lambda_{123}\right) \\
y_{1} y_{2}\left[r_{12} \lambda_{12}+2 r \lambda_{123}\right] & y_{2}^{2}\left(2 r_{12} \lambda_{123}\right) & y_{2}^{2} r \lambda_{12}
\end{array}\right) .
$$

where we have defined $\lambda_{12} \equiv \lambda_{1}+\lambda_{2}$ and $\lambda_{123} \equiv \lambda_{3}+\lambda_{1}-\lambda_{2}$. The following identifications are required to match Eq. (17) with Eq. (3).

$$
\begin{aligned}
a & \equiv y_{1}^{2} v^{2}\left[4 r_{12} \lambda_{123}+2 r \lambda_{12}\right]=y_{1}^{2} v^{2}\left[4 r_{12}\left(\lambda_{3}+\lambda_{1}-\lambda_{2}\right)+2 r\left(\lambda_{1}+\lambda_{2}\right)\right] \\
b & \equiv y_{2}^{2} v^{2} r \lambda_{12}=y_{2}^{2} v^{2} r\left(\lambda_{1}+\lambda_{2}\right) \\
c & \equiv y_{1} y_{2} v^{2}\left[r_{12} \lambda_{12}+2 r \lambda_{123}\right]=y_{1} y_{2} v^{2}\left[r_{12}\left(\lambda_{1}+\lambda_{2}\right)+2 r\left(\lambda_{3}+\lambda_{1}-\lambda_{2}\right)\right] \\
d & \equiv y_{2}^{2} v^{2}\left(2 r_{12} \lambda_{123}\right)=y_{2}^{2} v^{2}\left[2 r_{12}\left(\lambda_{3}+\lambda_{1}-\lambda_{2}\right)\right] .
\end{aligned}
$$

With the form of left-handed Majorana mass matrix for $\theta_{13}=0$ and $\theta_{23}=\pi / 4$ achieved within the model framework, let us now try to get the realistic mixing angles i.e., $\theta_{13} \neq 0$, deviations of $\theta_{23}$ from $\pi / 4$ as well as small corrections to the solar mixing. In order to get that, as already mentioned, we will shift from the condition $r_{11}=r_{22}=r$ by a small amount i.e., apply $r_{22}=r_{11}+\epsilon$ 
keeping $v_{1}=v_{2}=v$ fixed. The criterion $r_{22}=r_{11}+\epsilon$ makes $m_{R_{11}} \neq m_{R_{22}}$ in Eq. (11) causing small deviation from the maximal mixing between $N_{1 R}$ and $N_{2 R}$. Using $r_{22}=r_{11}+\epsilon$ with $v_{1}=v_{2}=v$ in Eq. (17) one can write $M_{v_{L}}^{\text {flavour }}$ as $M_{v_{L}}^{\text {flavour }}=M^{0}+M^{\prime}$, where $M^{0}$ is the dominant part and $M^{\prime}$ is a small contribution proportional to $\epsilon$. Thus,

$$
M^{0}=v^{2}\left(\begin{array}{ccc}
y_{1}^{2}\left[4 r_{12} \lambda_{123}+2 r_{11} \lambda_{12}\right] & y_{1} y_{2}\left[r_{12} \lambda_{12}+2 r_{11} \lambda_{123}\right] & y_{1} y_{2}\left[r_{12} \lambda_{12}+2 r_{11} \lambda_{123}\right] \\
y_{1} y_{2}\left[r_{12} \lambda_{12}+2 r_{11} \lambda_{123}\right] & y_{2}^{2} r_{11} \lambda_{12} & y_{2}^{2}\left(2 r_{12} \lambda_{123}\right) \\
y_{1} y_{2}\left[r_{12} \lambda_{12}+2 r_{11} \lambda_{123}\right] & y_{2}^{2}\left(2 r_{12} \lambda_{123}\right) & y_{2}^{2} r_{11} \lambda_{12}
\end{array}\right),
$$

and

$$
M^{\prime}=\epsilon\left(\begin{array}{ccc}
x & y & 0 \\
y & x^{\prime} & 0 \\
0 & 0 & 0
\end{array}\right)
$$

with

$$
\begin{aligned}
x & \equiv y_{1}^{2} v^{2} \lambda_{12}=y_{1}^{2} v^{2}\left(\lambda_{1}+\lambda_{2}\right) \\
x^{\prime} & \equiv y_{2}^{2} v^{2} \lambda_{12}=y_{2}^{2} v^{2}\left(\lambda_{1}+\lambda_{2}\right) \\
y & \equiv y_{1} y_{2} v^{2} \lambda_{123}=y_{1} y_{2} v^{2}\left(\lambda_{3}+\lambda_{1}-\lambda_{2}\right) .
\end{aligned}
$$

Again if we identify:

$$
\begin{aligned}
a^{\prime} & \equiv y_{1}^{2} v^{2}\left[4 r_{12} \lambda_{123}+2 r_{11} \lambda_{12}\right]=y_{1}^{2} v^{2}\left[4 r_{12}\left(\lambda_{3}+\lambda_{1}-\lambda_{2}\right)+2 r_{11}\left(\lambda_{1}+\lambda_{2}\right)\right] \\
b^{\prime} & \equiv y_{2}^{2} v^{2} r_{11} \lambda_{12}=y_{2}^{2} v^{2} r_{11}\left(\lambda_{1}+\lambda_{2}\right) \\
c^{\prime} & \equiv y_{1} y_{2} v^{2}\left[r_{12} \lambda_{12}+2 r_{11} \lambda_{123}\right]=y_{1} y_{2} v^{2}\left[r_{12}\left(\lambda_{1}+\lambda_{2}\right)+2 r_{11}\left(\lambda_{3}+\lambda_{1}-\lambda_{2}\right)\right] \\
d^{\prime} & \equiv y_{2}^{2} v^{2}\left(2 r_{12} \lambda_{123}\right)=y_{2}^{2} v^{2}\left[2 r_{12}\left(\lambda_{3}+\lambda_{1}-\lambda_{2}\right)\right]
\end{aligned}
$$

$M^{0}$ will be giving the form of Eq. (3). Let us define:

$$
\gamma \equiv\left(b^{\prime}-3 d^{\prime}-a^{\prime}\right) \text { and } \rho \equiv \sqrt{a^{\prime 2}+b^{\prime 2}+8 c^{\prime 2}+d^{\prime 2}-2 a^{\prime} b^{\prime}-2 a^{\prime} d^{\prime}+2 b^{\prime} d^{\prime}}
$$

and apply non-degenerate perturbation theory correct up-to first order and calculate the third first-order corrected ket:

$$
\left|\psi_{3}\right\rangle=\left(\begin{array}{c}
\frac{\epsilon}{\gamma^{2}-\rho^{2}}\left[\rho\left(\sqrt{2} y \cos 2 \theta_{12}^{0}-x^{\prime} \sin 2 \theta_{12}^{0}\right)-\gamma \sqrt{2} y\right] \\
-\frac{1}{\sqrt{2}}[1+\xi \epsilon] \\
\frac{1}{\sqrt{2}}[1-\xi \epsilon]
\end{array}\right)
$$

where,

$$
\xi \equiv\left[\gamma x^{\prime}+\rho\left(x^{\prime} \cos 2 \theta_{12}^{0}+\sqrt{2} y \sin 2 \theta_{12}^{0}\right)\right] /\left(\gamma^{2}-\rho^{2}\right) .
$$

One can compare Eq. (24) with the third column of the PMNS matrix in Eq. (1) and write:

$$
\sin \theta_{13}=\frac{\epsilon}{\gamma^{2}-\rho^{2}}\left[\rho\left(\sqrt{2} y \cos 2 \theta_{12}^{0}-x^{\prime} \sin 2 \theta_{12}^{0}\right)-\gamma \sqrt{2} y\right] .
$$

for a CP-conserving case that gives us non-zero $\theta_{13}$. From Eq. (24), we get the shift in $\theta_{23}$ from $\pi / 4$ as:

$$
\tan \varphi \equiv \tan \left(\theta_{23}-\pi / 4\right)=\xi \epsilon .
$$

The corrections to $\theta_{12}$ is given by:

$$
\tan \theta_{12}=\frac{\sin \theta_{12}^{0}+\epsilon \beta \cos \theta_{12}^{0}}{\cos \theta_{12}^{0}-\epsilon \beta \sin \theta_{12}^{0}}
$$

where,

$$
\beta \equiv \frac{\left[\frac{y}{\sqrt{2}} \cos 2 \theta_{12}^{0}+\frac{1}{2}\left(x-\frac{x^{\prime}}{2}\right) \sin 2 \theta_{12}^{0}\right]}{\rho} .
$$

Lepton flavour violating decays are completely forbidden in this model set-up owing to the $S 3$ symmetry. A detailed discussion can be found in [1]. 


\section{CONCLUSIONS}

In this radiative neutrino mass model based on $S 3 \times Z_{2}$ symmetry at one-loop level, we have two $Z_{2}$ odd right-handed neutrinos $N_{1 R}$ and $N_{2 R}$. When $N_{1 R}$ and $N_{2 R}$ are mixed maximally we get the left-handed Majorana neutrino mass matrix form needed for $\theta_{13}=0$ and $\theta_{23}=\pi / 4$ and solar mixing of any values corresponding to the popular lepton mixings like TBM, BM and GR as shown in Table 1. Deviating from maximal mixing between $N_{1 R}$ and $N_{2 R}$ yields realistic neutrino mixings in agreement with neutrino oscillation data i.e., non-zero $\theta_{13}$, shits of $\theta_{23}$ from $\pi / 4$ and small corrections to $\theta_{12}$. The lightest of the two $Z_{2}$ odd inert $S U(2)_{L}$ doublet scalars $\eta_{i},(i=1,2)$ can become a dark matter candidate. Thus the model is scotogenic.

\section{ACKNOWLEDGEMENTS}

SP is thankful to Prof. Amitava Raychaudhuri for important discussions. SP is supported in part by the National Science Centre (Poland) under the research Grant No. 2017/26/E/ST2/00470.

\section{References}

[1] S. Pramanick, Phys. Rev. D 100, no. 3, 035009 (2019) [arXiv:1904.07558 [hep-ph]].

[2] For the present status of $\theta_{13}$ see presentations from Double Chooz, RENO, Daya Bay, and T2K at Neutrino 2016 (http://neutrino2016.iopconfs.org/programme).

[3] M. C. Gonzalez-Garcia, M. Maltoni, J. Salvado and T. Schwetz, JHEP 1212, 123 (2012) [arXiv:1209.3023v3 [hep-ph]], NuFIT 3.2 (2018).

[4] D. V. Forero, M. Tortola and J. W. F. Valle, Phys. Rev. D 86, 073012 (2012) [arXiv:1205.4018 [hep-ph]].

[5] S. Pramanick and A. Raychaudhuri, Phys. Rev. D 88, no. 9, 093009 (2013) [arXiv:1308.1445 [hep-ph]]; S. Pramanick and A. Raychaudhuri, Phys. Lett. B 746, 237 (2015) [arXiv:1411.0320 [hep-ph]]; S. Pramanick and A. Raychaudhuri, Int. J. Mod. Phys. A 30, no. 14, 1530036 (2015) [arXiv:1504.01555 [hep-ph]]; S. Pramanick and A. Raychaudhuri, Phys. Rev. D 93, no. 3, 033007 (2016) [arXiv:1508.02330 [hep-ph]]; S. Pramanick and A. Raychaudhuri, Phys. Rev. D 94, no. 11, 115028 (2016) [arXiv:1609.06103 [hep-ph]]; S. Pramanick, Phys. Rev. D 98, no. 7, 075016 (2018) [arXiv:1711.03510 [hep-ph]].

[6] S. Pramanick, Nucl. Phys. B 963, 115282 (2021) [arXiv:1903.04208 [hep-ph]].

[7] S. Pramanick and A. Raychaudhuri, JHEP 1801, 011 (2018) [arXiv:1710.04433 [hep-ph]].

[8] E. Ma, Phys. Rev. D 73, 077301 (2006) [hep-ph/0601225]. 